\title{
The effect of the environmental parameter on the Hantavirus infection through a fractional-order SI model
}

\author{
S.Z. Rida ${ }^{1}$, A.S. Abdel Rady ${ }^{1}$, A.A.M. Arafa ${ }^{1 *}$, and M. Khalil ${ }^{2}$ \\ ${ }^{1}$ Department of mathematics, Faculty of Science, \\ South Valley University, Qena, Egypt \\ Email: szagloul@yahoo.com, safwat_ahmed@hotmail.com, anaszi2@yahoo.com \\ ${ }^{2}$ Department of mathematics, Faculty of Engineering, \\ Modern Science and Arts University (MSA), Giza, Egypt \\ Email: m_kh1512@yahoo.com
}

\begin{abstract}
In this paper, fractional-order model of the Hantavirus infection in terms of simple differential equations involving the mice population is presented. A study of the effect of changes in ecological conditions and diversity of habitats can be observed by varying the value of the environmental parameter $k$. Generalized Euler method (GEM) is considered in this paper to obtain an analytic approximate solution of this model.
\end{abstract}

Keywords: Generalized Euler method, Fractional order ordinary differential equations, Hantavirus fractional-order model.

\section{Introduction}

In 1993 an outbreak of a severe and unknown disease occurred in the North American Southwest, striking with mortality in excess of 50\%. The first reported cases came from healthy young adults who became sick and rapidly died at hospitals. Shortly afterwards, Sin Nombre virus (Bunyaviridae: Hantavirus), the first Hantavirus to be discovered in the New World, was identified as the infectious agent, and the very common deer mouse (Peromyscus maniculatus) as its host and reservoir [3]. Since then, numerous new Hantaviruses have been 
discovered throughout the Americas, each one hosted by a single mouse species, and many of them responsible for severe human pathology. Hantavirus pulmonary syndrome (HPS) is an increasingly recognized infectious disease associated with infection of humans by new world Hantaviruses. The disease was transmitted to people by contact with rodents [7]. This leads to an extensive effort to trap all different types of rodents within the Four Corners area and detect the rodent with the antibodies to the strain of Hantavirus in question. Among all types of rodents trapped, the deer mouse was found to be the principle reservoir to the previously unknown strain of Hantavirus. The deer mouse population is abundant in North America where a potential for extensive outbreaks of HPS exists. The deer mouse is found in rural and semi-rural areas, in barns, homes and other buildings. Researchers believe that the virus is being passed from the deer mouse to humans from the contact made in these settings. Approximately $25 \%$ of the deer mice trapped were found to be infected with Hantavirus. Other mice were also found to be infected, but in lesser quantities [2]. The infection does not produce any clinical manifestation in the mice, which are asymptomatic carriers. Wild populations of host mice usually harbor a varying proportion of infected animals [3]. They are mostly adult males, and typically the most battered ones, from which it has been conjectured that contagion takes place by direct contact during fights. Infected females transmit their antibodies to newborns, which are thus protected from infection until they are weaned and become susceptible. Infected mice, it also seems, remain infected and infectious for their whole life (wild mice have short lives, with the luckier ones living a couple of years). It has been observed that the outbreaks of HPS in the North American Southwest in 1993 and again in 1998-2000 were associated with the El Nino-Southern Oscillation (ENSO) phenomenon [1]. El Nino-the warm phase of the ENSO is accompanied by increased fall-spring precipitation in the arid and semi-arid regions of New Mexico and Arizona, in turn initiating a greater production of food resources for rodents: seeds, berries, nuts, insects. A "trophic cascade" is triggered, which leads to greater reproduction in rodents and, within a year, to large increases in rodent densities. At these higher densities, rodents disperse across the landscape ("ratadas," as they are called in Latin American countries), and come into contact with humans in homes and businesses. An increase in the density of infected rodents was observed one year after the peak rodent densities, suggesting that a "wave" of virus infection was following the "wave" of rodent dispersal [4]. During times of adverse environmental conditions, a complete disappearance of the disease from local populations has been observed, as well as its eventual reappearance when conditions change.

The rest of the paper is organized as follows. In section 2, a discussion about the basic system of the Hantavirus infection is presented. Section 3 presents the importance of fractional calculus. Generalized Taylor's formula and Generalized Euler method are introduced in section 4 and 5 respectively. Section 6 is devoted to the numerical results of the presented problem. 


\section{A basic system of the Hantavirus infection}

To mathematically describe the full dynamics of the biological system that affects the mice population, the humans, the virus and the environment is not an easy mission. There are two major characteristics of the infection which are derived from the fact that environmental conditions play an important role in the dynamics of the disease. The first is the temporal attribute, whereby infection can completely die out from a population of mice if environmental conditions are poor, and then to reappear intermittently or when the conditions change $[3,13]$. The second is the spatial attribute, where there are signs of "focality" of the infection in "reservoir" populations. As environmental conditions change, these "refugia" of the reservoir can expand or contract, carrying the infection to other places. Abramson and Kenkre [2] introduced a mathematical model that incorporates decay by death of the mice population, the increase by birth and effect of the environment to stabilize the population, and their movement by diffusion. We shall only perform our study on the model which integrates the temporal characteristics, omitting the diffusion process. Abramson and Kenre [2] proposed a basic model of a biological system that mimics the spread of the Hantavirus infection which studies its temporal behaviour. The system consists of a population of susceptible and infected mice, represented by $m_{s}$ and $m_{i}$ respectively. It is noted that the sex and age composition of the population are disregarded in this model. Eqs. (1) and (2) show the contagion of the infection, that converts susceptible into infected:

$$
\begin{aligned}
& \frac{d m_{s}}{d t}=b m-c m_{s}-\frac{m_{s} m}{k}-a m_{s} m_{i} \\
& \frac{d m_{i}}{d t}=-c m_{i}-\frac{m_{i} m}{k}+a m_{s} m_{i}
\end{aligned}
$$

Where $m(t)=m_{s}(t)+m_{i}(t)$ is the total population of mice. The motivation for the terms in (1) and (2) follows:

Births: $b m$ represents births of mice, all of them born susceptible, at a rate proportional to the total density, since all mice contribute equally to the procreation [13].

Deaths: $c$ represents the rate of population decay by death, proportional to the corresponding density. If necessary, separate rates $c_{s}$ and $c_{i}$ could be introduced for the susceptible and infected populations respectively. For Hantavirus, which does not seem to alter any physiological parameter of their hosts, we have the same death rate for both susceptible and infected mice.

Competition: $-m_{s, i} m / k$ represents a limitation process in the population growth, due to competition for shared resources. Each is proportional to the probability of an encounter of a pair formed by one mouse of the corresponding class, susceptible or infected, and one mouse of any class (since every mouse, either susceptible or infected, has to compete with the whole population). $k$ is $a$ 
"carrying capacity," characterizing in a simplified way the capacity of the medium to maintain a population of mice. Higher values of carrying capacity represent a higher availability of water, food, shelter and other resources that mice can use to thrive [13].

Infection: $a m_{s} m_{i}$ represents the number of susceptible mice that get infected, due to an encounter with an infected (and consequently infectious) mouse, at a rate $a$ that we assume constant. Contagion between deer mice is believed to take place during direct animal contact, mainly by biting. There are four parameters that characterize the system in (1) and (2), which are $a, b, c$ and $k$. Of those, $k$ is chosen to be the control parameter of the dynamics because it best represents the influence of the environment. Changes in ecological conditions and diversity of habitats can be observed by varying the value of $k$. Although the system has four equilibria, two of them are irrelevant to the analysis, that is, the null state (very unstable) and a state with $m_{i}<0$ for any parameters. The other two equilibria interchange their stability character at a critical value of the carrying capacity, which is given as

$k_{c}=\frac{b}{a(b-c)}$

More elaborate models could incorporate a density dependence on a, for example due to an increased frequency of fights when the density is too high and the population feels overcrowded [2,3]. Since the infection is chronic, infected mice do not die of it, and infected mice do not lose their infectiousness probably for their whole life, this single term adequately describes the infection dynamics of the two subpopulations. The presented model is able to successfully explain several field observations as environmentally controlled phase transitions, thus providing an analytical support to biological hypotheses such as the trophic cascade. According to [3], the equations (1) and (2) reflect a continuous model but in a real system, the population is discrete where it vanishes if it drops below one. The zero population state, which may be unstable in the continuous perspective, is stable against infinitesimal perturbations in a discrete model. In reality, a system requires a finite perturbation of one full mouse to start moving toward the positive equilibrium. It is only sensible that an infection that has died off will only reappear when an infected mouse has entered the system from the outside.

This paper attempts to derive a numerical solution for fractional order equations of Hantavirus infection model of order $\alpha_{1}, \alpha_{2}>0$

$$
\begin{aligned}
& D^{\alpha_{1}}\left(m_{s}\right)=b m-c m_{s}-\frac{m_{s} m}{k}-a m_{s} m_{i}, \\
& D^{\alpha_{2}}\left(m_{i}\right)=-c m_{i}-\frac{m_{i} m}{k}+a m_{s} m_{i}
\end{aligned}
$$

The reason of using fractional order differential equations (FOD) is that FOD are naturally related to systems with memory which exists in most biological systems. 
Also they are closely related to fractals which are abundant in biological systems. The results derived of the fractional system (3), (4) are of a more general nature.

\section{Fractional calculus}

Although fractional derivatives have a long mathematical history [8], for many years they were not used in biology or physics. One possible explanation of such unpopularity could be that there are multiple nonequivalent definitions of fractional derivatives [9]. Another difficult is that fractional derivatives have no evident geometrical interpretation because of their nonlocal character [10]. It was found that various; especially interdisciplinary applications can be elegantly modeled with the help of the fractional derivatives. For example, the nonlinear oscillation of earthquake can be modeled with fractional derivatives, and the fluiddynamic traffic model with fractional derivatives can eliminate the deficiency arising from the assumption of continuum traffic flow. However, during the last ten years fractional calculus starts to attract much more attention of physicists and mathematicians [17]. In biology, it has been deduced that the membranes of cells of biological organism have fractional-order electrical conductance [4] and then are classified in groups of non-integer order models. FODE are naturally related to systems with memory which exists in most biological systems. Also, they are closely related to fractals, which are abundant in biological systems [10,11]. We first give the definition of fractional-order integration and fractional-order differentiation [14]. For the concept of fractional derivative, we will adopt Caputo's definition, which is a modification of the Riemann-Liouville definition and has the advantage of dealing properly with initial value problems.

Definition 1 The fractional integral of order $\alpha>0$ of a function $f: R^{+} \rightarrow R$ is given by

Hence we have

$$
\begin{gathered}
J^{\alpha} f(x)=\frac{1}{\Gamma(\alpha)} \int_{0}^{x}(x-t)^{\alpha-1} f(t) d t, \quad \alpha>0, x>0, \\
J^{0} f(x)=f(x) .
\end{gathered}
$$

$$
J^{\alpha} t^{\gamma}=\frac{\Gamma(\gamma+1)}{\Gamma(\alpha+\gamma+1)} t^{\alpha+\gamma}, \quad \alpha>0, \gamma>-1, t>0
$$

Definition 2 Riemann-Liouville and Caputo fractional derivatives of order $\alpha$ of a continuous function $f: R^{+} \rightarrow R$ is given respectively by

$$
\begin{aligned}
& D_{*}^{\alpha} f(x)=D^{m}\left(J^{m-\alpha} f(x)\right), \\
& D^{\alpha} f(x)=J^{m-\alpha}\left(D^{m} f(x)\right),
\end{aligned}
$$


Where

$$
m-1<\alpha \leq m, m \in N \text {. }
$$

The definition of fractional derivative involves an integration which is non-local operator (as it is defined on an interval) so fractional derivative is a non-local operator. In other word, calculating time-fractional derivative of a function $f(t)$ at some time $t=t_{1}$ requires all the previous history, i.e. all $f(t)$ from $t=0$ to $t=t_{1}$.

\section{Generalized Taylor's formula}

In this section we introduce a generalization of Taylor's formula that involves Caputo fractional derivatives. This generalization is presented in [19].

Suppose that $D_{*}^{k \alpha} f(x) \in C(0, a]$ for $k=0,1, \ldots, n+1$, where $0<\alpha \leq 1$. So we have

$f(x)=\sum_{i=0}^{n} \frac{x^{i \alpha}}{\Gamma(i \alpha+1)}\left(D_{*}^{i \alpha}\right)(0+)+\frac{\left(D_{*}^{(n+1) \alpha} f\right)(\xi)}{\Gamma((n+1) \alpha+1)} x^{(n+1) \alpha}$

With $0 \leq \xi \leq x, \forall x \in(0, a]$.

In case of $\alpha=1$, the generalized Taylor's formula (5) reduces to the classical Taylor's formula.

\section{Generalized Euler method (GEM)}

Most nonlinear fractional differential equations do not have analytic solutions, so approximations and numerical techniques must be used [15]. The decomposition method (ADM) and the variational iteration method (VIM) are relatively new approaches to provide an analytical approximate solution to linear and nonlinear problems, and they are particularly valuable as tools for scientists and applied mathematicians, because they provide immediate and visible symbolic terms of analytic solutions, as well as numerical approximate solutions to both linear and nonlinear differential equations. In recent years, the application of the ADM, VIM, $[15,16]$ in linear and nonlinear problems has been developed. On the other hand, these methods are effective for small time, i.e. $t<<1$, however the standard homotopy perturbation method (HPM) cannot solve the problem for larger time and in fact the solution of the chaotic system using HPM is an open problem [16]. Nevertheless by chance, there are cases at which these methods give good approximation for a large range of time $(t)$ [15]. A few numerical methods for fractional differential equations have been presented in the literature $[9,12]$. However many of these methods are used for very specific types of differential equations, often just linear equations or even smaller classes. In [19], Odibat and Momani derived the generalized Euler's method that we have developed for the numerical solution of initial value problems with Caputo 
derivatives. The method is a generalization of the classical Euler's method. Consider the initial value problem $[5,6]$.

$D_{*}^{\alpha} y(t)=f(t, y(t)), y(0)=y_{0}, 0<\alpha \leq 1$,

Let $[0, a]$ be the interval over which we want to find the solution of the problem (6). In actuality, we will not find a function $y(t)$ that satisfies the initial value problem (6). Instead, a set of points $\left\{\left(t_{j}, y\left(t_{j}\right)\right\}\right.$ is generated, and the points are used for our approximation. For convenience we subdivide the interval $[0, a]$ into $k$ subintervals $\left[t_{j}, t_{j+1}\right]$ of equal width $h=a / k$ by using the nodes $t_{j}=j h$, for $j$ $=0,1, \ldots, k$. Assume that $y(t), D_{*}^{\alpha} y(t)$ and $D_{*}^{2 \alpha} y(t)$ are continuous on $[0, a]$ and use the generalized Taylor's formula (5) to expand $y(t)$ about $t=t_{0}=0$. For each value $t$ there is a value $c_{1}$ so that

$y(t)=y\left(t_{0}\right)+\left(D_{*}^{\alpha} y(t)\right)\left(t_{0}\right) \frac{t^{\alpha}}{\Gamma(\alpha+1)}+\left(D_{*}^{2 \alpha} y(t)\right)\left(c_{1}\right) \frac{t^{2 \alpha}}{\Gamma(2 \alpha+1)}$

When $\left(D_{*}^{\alpha} y(t)\right)\left(t_{0}\right)=f\left(t_{0}, y\left(t_{0}\right)\right)$ and $h=t_{1}$ are substituted into equation (7), the result is an expression for $y\left(t_{1}\right)$ :

$y\left(t_{1}\right)=y\left(t_{0}\right)+f\left(t_{0}, y\left(t_{0}\right)\right) \frac{h^{\alpha}}{\Gamma(\alpha+1)}+\left(D_{*}^{2 \alpha} y(t)\right)\left(c_{1}\right) \frac{h^{2 \alpha}}{\Gamma(2 \alpha+1)}$

If the step size $h$ is chosen small enough, then we may neglect the second-order term (involving $h^{2 \alpha}$ ) and get

$y\left(t_{1}\right)=y\left(t_{0}\right)+\frac{h^{\alpha}}{\Gamma(\alpha+1)} f\left(t_{0}, y\left(t_{0}\right)\right)$

The process is repeated and generates a sequence of points that approximates the solution $y(t)$. The general formula for generalized Euler's method (GEM) when $t_{j+1}=t_{j}+h$ is

$y\left(t_{j+1}\right)=y\left(t_{j}\right)+\frac{h^{\alpha}}{\Gamma(\alpha+1)} f\left(t_{j}, y\left(t_{j}\right)\right)$

for $j=0,1, \ldots, k-1$. It is clear that if $\alpha=1$, then the generalized Euler's method (8) reduces to the classical Euler's method.

\section{Results and discussions}

Using the constants mentioned earlier, we shall vary the initial values of $m_{s}$ and $\mathrm{m}_{\mathrm{i}}$ against different values of parameter $\mathrm{k}$. This gives us an appreciation of the effect of initial values and $k$ on the system. The time step $h$ used in this paper is 0.1 . We shall use the constants $a=0.1, b=1, c=0.5$, as were chosen by Abramson and Kenkre [3]. In this case $k_{c}=20$. 


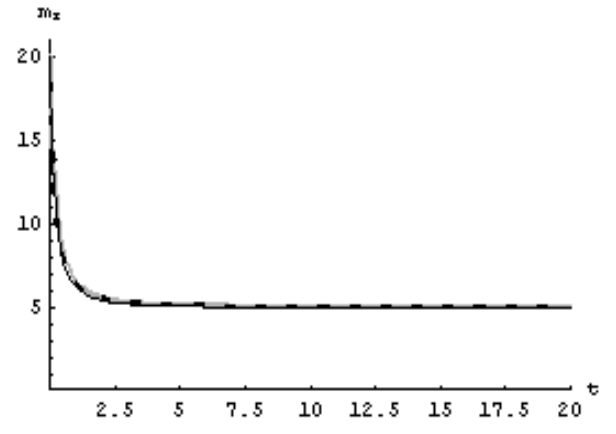

(a)

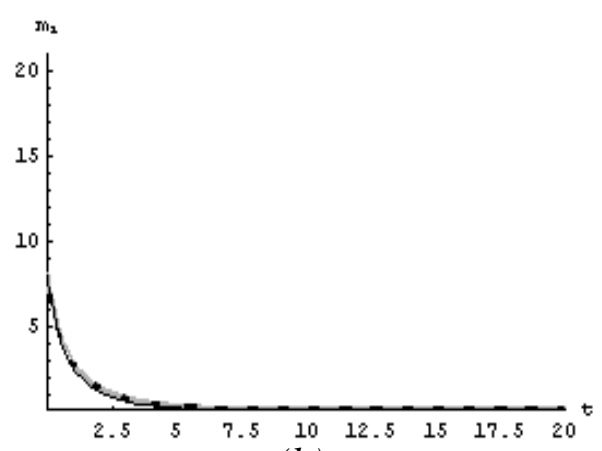

(b)

Figure 1: The populations of susceptible $(a)$ and infected $(b)$ mice versus time in years for $k=10$ : Gray solid line $(\alpha=1)$, Dotted line $(\alpha=0.99)$, Black solid line $(\alpha=0.95)$.

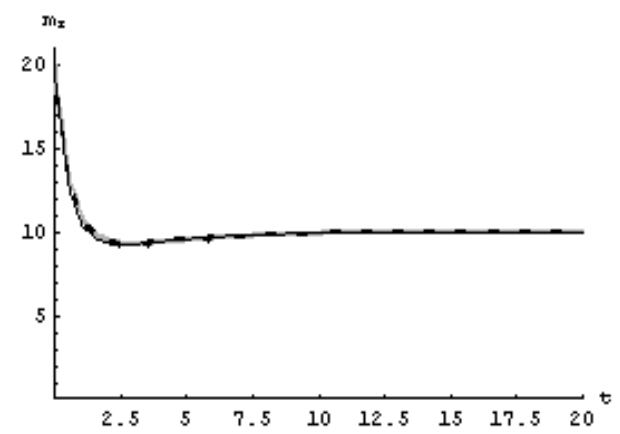

(a)

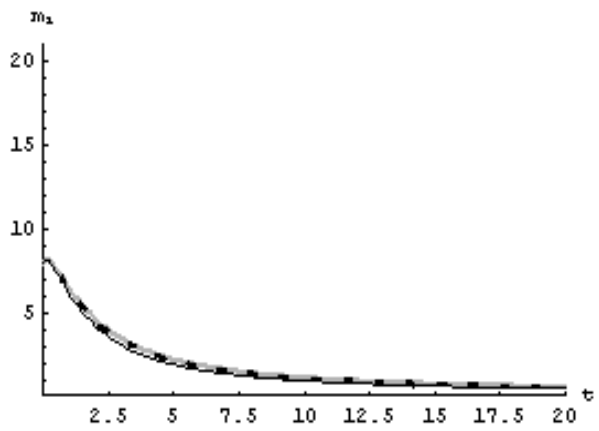

(b)

Figure 2: The populations of susceptible $(a)$ and infected $(b)$ mice versus time in years for $k=20$ : Gray solid line $(\alpha=1)$, Dotted line $(\alpha=0.99)$, Black solid line $(\alpha=0.95)$. 


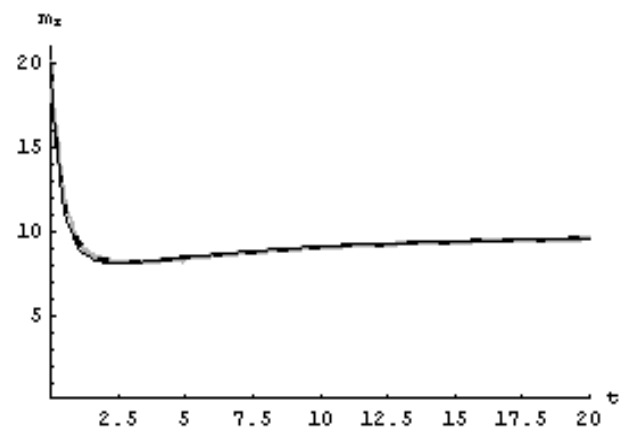

(a)

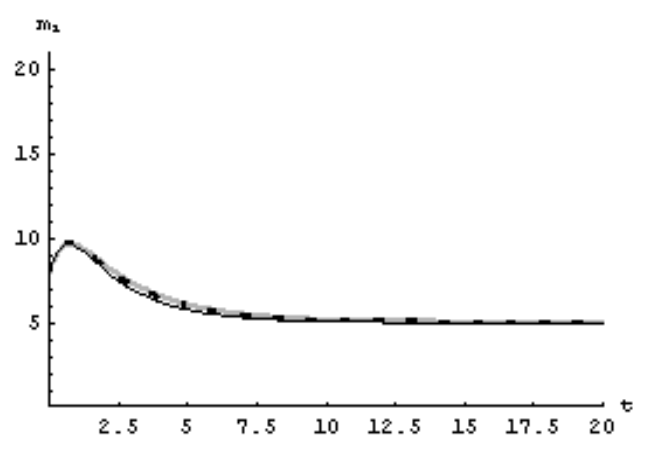

(b)

Figure 3: The populations of susceptible $(a)$ and infected $(b)$ mice versus time in years for $k=30$ : Gray solid line $(\alpha=1)$, Dotted line $(\alpha=0.99)$, Black solid line $(\alpha=0.95)$.

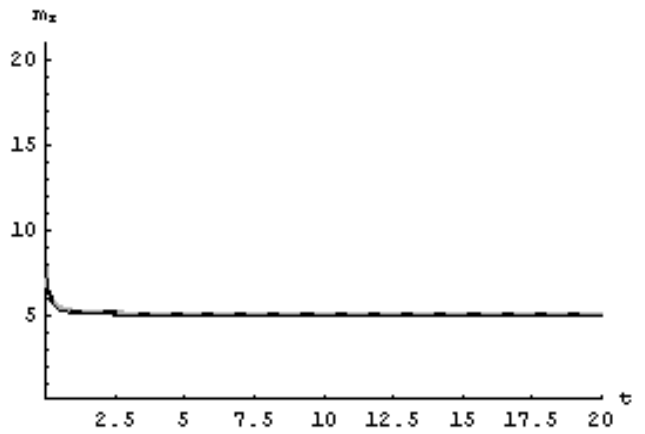

(a)

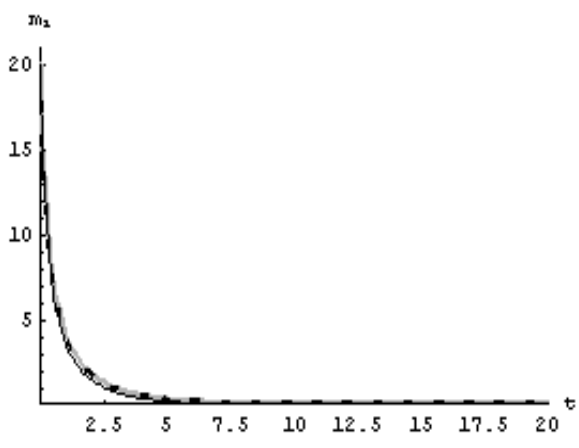

(b)

Figure 4: The populations of susceptible $(a)$ and infected $(b)$ mice versus time in years for $k=10$ : Gray solid line $(\alpha=1)$, Dotted line $(\alpha=0.99)$, Black solid line $(\alpha=0.95)$. 


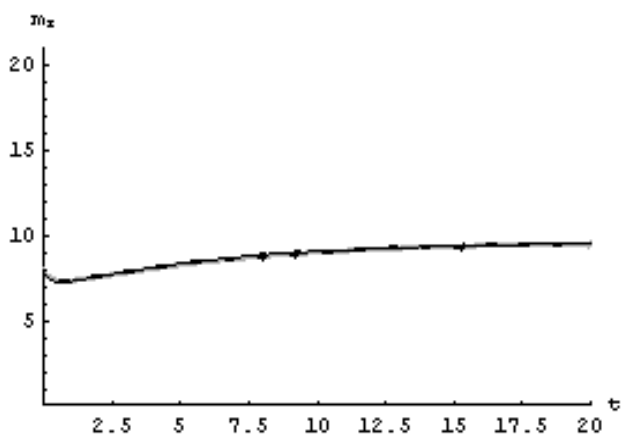

(a)

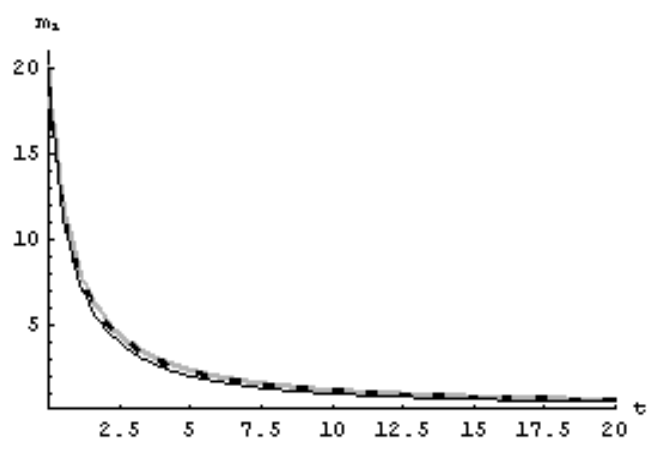

(b)

Figure 5: The populations of susceptible $(a)$ and infected $(b)$ mice versus time in years for $k=20$ : Gray solid line $(\alpha=1)$, Dotted line $(\alpha=0.99)$, Black solid line $(\alpha=0.95)$.

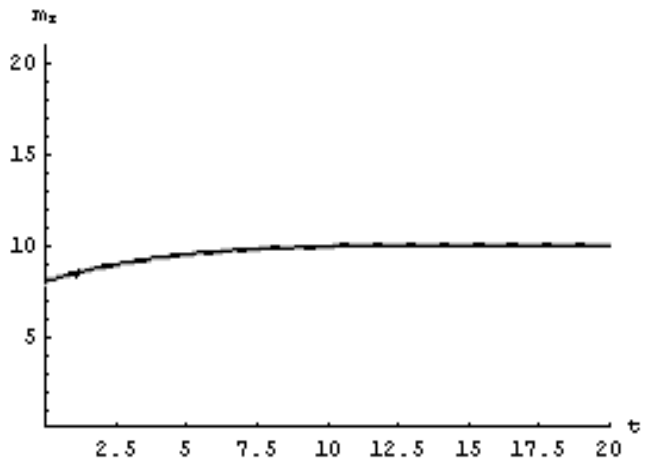

(a)

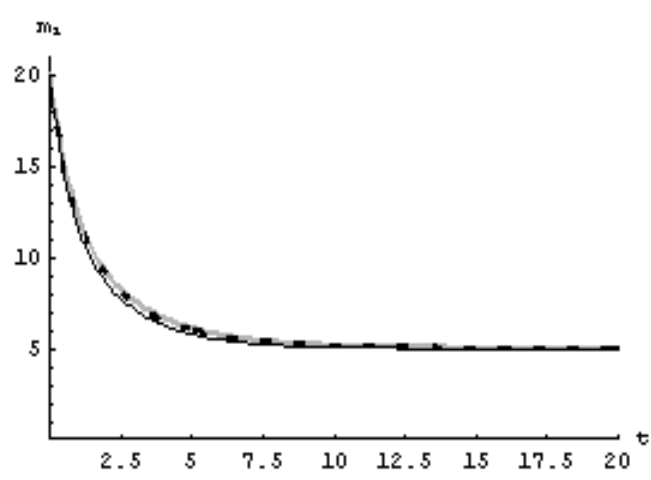

(b)

Figure 6: The populations of susceptible $(a)$ and infected $(b)$ mice versus time in years for $k=30$ : Gray solid line $(\alpha=1)$, Dotted line $(\alpha=0.99)$, Black solid line $(\alpha=0.95)$.

\section{Conclusion}

In this paper, generalized Euler method (GEM) was implemented to describe the effect of carrying capacity $k$, which is used as a control parameter in the model of HPS described by the presented fractional model. The results show that the solution continuously depends on the time-fractional derivative and on the values of the parameters. The presented figures show the numerical solutions for (3), (4) with varying values of $m_{s}(0)$ and $m_{i}(0)$ against different values of $k$. In Figures (1-3), we assumed that $m_{s}(0)=20, m_{i}(0)=8$, but in Figures (4-6), we assumed that $m_{s}(0)=8, m_{i}(0)=2$. As mentioned before, changes in ecological conditions and diversity of habitats affect the mice population which can be seen 
through the carrying capacity $k$. Figure 1 , and Figure 4 show that if $k<k_{c}$, the population of infected mice $m_{i}$, will reduce to zero (after about 5 years) regardless of the initial number. In other words, for all initial values, the infection will die away. The population of susceptible mice $m_{s}$ on the other hand, will approach a steady state of 5. For $k=k_{c}$, in Figure 2, and Figure 5, the number of infected mice, $m_{i}$ will also decrease to zero, but at a much slower rate and $m_{s}$ will reach a steady state of about 10 years. Again, this observation is for all the set of initial values used. In Figure 3, and Figure $6,>k_{c}$, then for all sets of initial values, $m_{s}$ will approach a steady state of 10 whereas $m_{i}$ will maintain at 5 . We noticed here that the infected population $m_{i}$, does not shrink to zero like for the other two environmental conditions. This corresponds to the notion that infected mice will "thrive" when the environmental conditions are favorable.

As a definition of fractional calculus: $\lim _{\alpha \rightarrow 1} D^{\alpha} f(t)=D f(t)$ has been provided. In the presented problem, the susceptible group $m_{s}$, the infected group $m_{i}$, and the removed group, have been obtained, the results obtained show that when $\alpha \rightarrow 1$ the solution of the fractional model, $\left(m_{s}\right)_{\alpha}$ and $\left(m_{i}\right)_{\alpha}$, reduce to the standard solution $m_{s}$ and $m_{i}$.

In this paper, we modified the ODE of Hantavirus model into a system of fractional-order. Our studies on the use of GEM for solving the presented model show that GEM is a good tool in solving the biological system. One of the advantages of GEM is its capability of presenting us with continuous solutions, thus giving us better understanding, insight as well as detail over the time interval.

\section{References}

[1] G. Abramson, V.M. Kenkre, Spatio-temporal patterns in the Hantavirus infection, Phys. Rev. E 66 (2002) 011912.

[2] G. Abramson, V.M. Kenkre, T.L. Yates, B.R. Parmenter, Traveling waves of infection in the Hantavirus epidemics, Bull. Math. Biol. 65 (2003) 519-534.

[3] G. Abramson, Mathematical modeling of Hantavirus : From the mean field to the individual level, Progress in Mathematical Biology Research, Nova Science Publishers, Inc. (2007).

[4] Aguirre, M.A., Abramson, G., Bishop, A.R. and Kenkre, V.M., Simulations in the mathematical modeling of the spread of the Hantavirus, Phys. Rev. E 66, 041908 (2002).A.H. Nayfeh, Perturbation Methods, John Wiley, New York, 1973.

[5] A.A.M. Arafa, S.Z. Rida and M. Khalil, Fractional modeling dynamics of HIV and CD4+ T-cells during primary infection, Nonlinear Biomedical Physics 6( 2012) 1-7. 
[6] A.A.M. Arafa, S.Z. Rida and M. Khalil, Fractional Order Model of Human T-cell Lymphotropic Virus I (HTLV-I) Infection of CD4+T-cells, Advanced Studies in Biology, 3(2011) 347 - 353.

[7] S. Busenberg, P. van den Driessche, Analysis of a disease transmission model in a population with varying size, J. Math. Biol. 28 (1990) 257-270.

[8] K.S. Cole, Electric conductance of biological systems, in: Proc. Cold Spring Harbor Symp. Quant. Biol, Cold Spring Harbor, New York, (1993) 107-116.

[9] K. Diethelm and G. Walz, Numerical solution for fractional differential equations by extrapolation, Numerical algoritms ,16 (1997) 231-253.

[10] A.M.A. El-Sayed, S.Z. Rida, A.A.M. Arafa, On the Solutions of Timefractional Bacterial Chemotaxis in a Diffusion Gradient Chamber, International Journal of Nonlinear Science , 7(2009) 485-492.

[11] A.M.A. El-Sayed, S.Z. Rida, A.A.M. Arafa, Exact Solutions of FractionalOrder Biological Population Model, Commun. Theor. Phys. 52 (2009) 992996.

[12] A.M.A. El-Sayed, A. E. M. El-Mesiry, and H. A. A. El-Saka, Numerical solution for multi-term fractional (arbitrary) orders differential equations, Comput. Appl. Math., 23(2004)33-54.

[13] S.M. Goh, A.I.M. Ismail, M.S.M. Noorani, I. Hashim, Dynamics of the Hantavirus infection through variational iteration method, Nonlinear Analysis: Real World Applications, 10 (2009) 2171-2176.

[14] I. Hashim, O. Abdulaziz, S. Momani, Homotopy analysis method for fractional IVPs, Communications in Nonlinear Science and Numerical Simulation 14 (2009) 674-684.

[15]O.D. Makinde, Adomian decomposition approach to a SIR epidemic model with constant vaccination strategy, App. Math. comput., 184 (2007) 842848.

[16] Mehmet Merdan, Homotopy perturbation method for solving a model for HIV infection of $\mathrm{CD}^{+} \mathrm{T}$ cells, İstanbul Ticaret Üniversitesi Fen Bilimleri Dergisi, 6 (2007) 59-62.

[17] S.Z. Rida, H.M. El-Sherbiny, A.A.M. Arafa, On the solution of the fractional nonlinear Schrödinger equation, Physics Letters A, 372 (2008) 553-558.

[18] Z. Odibat and N. Shawagfeh, Generalized Taylor's formula, Appl. Math. Comput. 186 (2007) 286-293. 